\title{
Les mots d'autrui, leur mise en texte, leurs publics
}

\section{Bertrand Masquelier}

\section{(2) OpenEdition}

\section{Journals}

Édition électronique

URL : https://journals.openedition.org/clo/2393

DOI : $10.4000 /$ clo.2393

ISSN : 2266-1816

Éditeur

INALCO

\section{Édition imprimée}

Date de publication : 31 décembre 2015

ISBN : 9782858312276

ISSN : 0396-891X

\section{Référence électronique}

Bertrand Masquelier, «Les mots d'autrui, leur mise en texte, leurs publics », Cahiers de littérature orale [En ligne], 77-78 | 2015, mis en ligne le 10 mai 2016, consulté le 01 juillet 2021. URL : http:// journals.openedition.org/clo/2393; DOI : https://doi.org/10.4000/clo.2393

\section{(c) (7) \&}

Cahiers de littérature orale est mis à disposition selon les termes de la Licence Creative Commons Attribution - Pas d'Utilisation Commerciale 4.0 International. 


\title{
Les mots d'autrui, leur mise en texte, leurs publics
}

\author{
Bertrand MASQuelier \\ Lacito, Sorbonne Paris Cité
}

\section{Entendre des paroles de calypso, ici et là}

Paris, septembre 2013, conversation avec un linguiste haïtien : nous sommes assis à une table de l'une des brasseries qui jouxtent le Forum des Halles. Nos échanges portent sur le konpa (haïtien) et le calypso (trinidadien), deux styles de chanson, comme de musique. Au regard des classifications des musicologues, ces styles témoignent des « cultures » ou « traditions » musicales de ces îles (Haïti et Trinidad ${ }^{1}$ ); ils sont étroitement liés à l'histoire sociale (coloniale et postcoloniale) de ces lieux.

Plus spécifiquement, notre dialogue porte sur l'étude des performances publiques de ces chansons. Nos arguments se veulent critiques de toute analyse qui serait fondée exclusivement sur des textes-transcriptions et des partitions. Notre commentaire, en un sens, ne fait que revisiter le changement de point de vue intervenu depuis les années 1960, quand prédominait l'analyse structurale. L'anthropologie linguistique et l'ethnomusicologie contemporaines privilégient désormais l'étude (et l'observa-

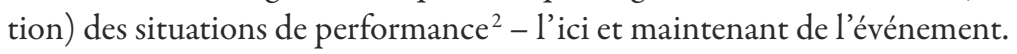

1. L'expression Trinidad renvoie ici, et pour la suite de cet essai, à l'ensemble social et politique que constituent les deux îles réunies de la République de Trinidad-et-Tobago.

2. Le terme de performance est à entendre dans un sens large. Il couvre les phénomènes sociaux et culturels spectaculaires, qu' ils soient scéniques, rituels, ou non. Le lecteur peut se reporter aux travaux de quelques précurseurs : parmi eux, Victor Turner en anthropologie, Erving Goffman en sociologie et Richard Schechner en études théâtrales. Pour les travaux en ethnomusicologie dans la perspective de l'ethnopoétique contemporaine, voir CALAme et al (2010). Sur le tournant performatif en anthropologie sociale et linguistique, voir MASQUELIER (2014). 
Dans l'espace caribéen ${ }^{3}$, les enregistrements de terrain (field-recordings), de musiques (instrumentales) et de chants sont abondants. Dans la plupart des cas, la qualité de ces enregistrements permet, à l'auditeur attentif, d'accéder à quelques indices sonores susceptibles de révéler le caractère public ${ }^{4}$, spontané ou « naturel » des circonstances de la performance; dans le meilleur des cas, ce même auditeur est en mesure de saisir quelque chose de l'interaction entre chanteur, musiciens et auditoire. Les plus explicites de ces indices sont des directives données par le chanteur aux musiciens, des commentaires qui émanent des musiciens à l'égard du chanteur, des échanges collusifs discrets entre le chanteur et son auditoire, voire des improvisations du chanteur en cours de performance. L'enregistrement est envisagé dès lors pour ce qu'il dévoile de l'événement situé dans le moment de son occurrence (bic et nunc). De ce point de vue, l'anthropologue linguiste et l'ethnomusicologue se trouvent dans une position qui n'est pas sans rappeler celle du spécialiste de l'antiquité grecque ou romaine, lorsque ce dernier tente de restituer, pour le lecteur contemporain, le dispositif énonciatif, actionnel, pragmatique, qui innerve les textes anciens ou fragments de textes, de poésie ou de théâtre (Calame, 2015).

En privilégiant l'étude des usages du langage en situation d'interaction (de communication), l'anthropologie linguistique s'est focalisée sur les pratiques associées à des lieux et des moments spécifiques (setting), bornés dans le temps et l'espace (situations, scenes). Est-il possible d'étendre le champ de l'enquête, de varier l'échelle de la description et de l'analyse ? L'histoire sociale (entendue dans un sens large) des chansons de calypso et la documentation sonore et textuelle qui lui est associée suggèrent quelques pistes d'enquêtes nouvelles.

Tout au long du $\mathrm{xx}^{\mathrm{e}}$ siècle, le calypso est resté indissociable de la célébration du carnaval. Au fil des décennies, ses chanteurs (et compositeurs) l'ont adapté dans un esprit de créativité pour en proposer différents styles. Les innovations

3. Les principaux enregistrements ethnomusicologiques, réalisés par Alan Lomax et Emory Cook, ont été effectués à Trinidad dans les années 1950 et 1960. Les premiers enregistrements de calypsos de l'industrie new-yorkaise du disque datent, quant à eux, de 1914.

4. Le caractère public que nous attribuons ici à ces performances enregistrées restitue ce que l'expression (métaphorique) de l'anglais live recording cherche à dire. Si les règles des « jeux de langage » de la performance scénique, « publique », permettent de la distinguer - qu'elle soit ou non enregistrée - d'un enregistrement de studio destiné à un public, il y a bien (acte de) performance dans les deux situations. Il faut souligner en effet l'extrême attention qui, dans ces circonstances spécifiques de production en studio, est portée à la performance et aux effets recherchés. 
ont porté tout à la fois sur ce qui était dit en chanson, ou pouvait l'être, et leurs formes musicales. Les chanteurs ne pouvaient ignorer les changements de leur environnement musical, qu'il soit local ou global. Il leur fallait anticiper, parfois surprendre, les attentes de leur auditoire. Mais pour ces chanteurs, en tant que membres de la société trinidadienne, dans la plupart des cas de sa classe populaire et défavorisée, il s'agissait de mettre en mots (aussi bien qu'en musique) l'expérience vécue de leur situation sociale, de rendre compte des événements du moment, année après année, de l'histoire, à petite échelle le plus souvent, de la communauté trinidadienne dans son ensemble et de ses transformations. Le calypso est ainsi devenu un mode de publication, textuel et musical, des faits qui affectent la vie quotidienne des Trinidadiens. Sous bien des aspects, même les plus anodins, il est une manière de dire éminemment politique. Si bien que l'historiographie, au fil du temps des diverses formes (textuelles et musicales) du calypso, s'avère être d'une étonnante complexité.

Certaines périodes sont remarquables pour les ruptures qui s'y manifestent. Ainsi, depuis les années 1930 à Trinidad, les performances scéniques des calypsos ne sont plus exclusives de la seule période du carnaval comme c'était le cas auparavant, depuis la fin du XIX ${ }^{\mathrm{e}}$ siècle. Accessibles tout au long de l'année, les performances se tiennent en de nombreux lieux, notamment là où touristes et visiteurs étrangers s'agrègent. En raison de l'expatriation d'une poignée de chanteurs trinidadiens vers New York, les performances de calypso, dès les années 1920 et 1930, furent accueillies dans certains des clubs de Harlem et du sud de Manhattan. Dans les années 1950, l'émigration massive de ressortissants des colonies britanniques des Caraïbes vers la Grande-Bretagne, Londres en particulier, fut l'une des voies de la diffusion du calypso. Ces mêmes années 1950 sont remarquables pour l'ampleur de l'intérêt qui lui sera porté, une passion que la presse américaine va qualifier de calypso-folie (calypso craze). Dans les années 1940-1950, il s'est introduit définitivement au sein d'un espace de publics hétérogènes. L'engouement ne se limite désormais plus aux frontières des communautés d'origine africaine des grandes villes nord-américaines, des Caraïbes, ou de l'Europe ; il concerne tous les publics. Les succès commerciaux de l'album intitulé Calypso de Harry Belafonte en 1955 et celui de l'acteur Robert Mitchum (un album de 1957, intitulé Calypso, is like so ${ }^{5}$ ) confirment l'extension de la réception des cultures musicales caribéennes. Quelques productions cinématographiques hollywoodiennes de cette même période y

5. L'album de R. Mitchum de 1957 contient plusieurs reprises de « classiques » locaux, trinidadiens. 
ajouteront leurs intrigues caribéennes ${ }^{6}$, leurs images des lieux, leurs versions européanisées des musiques locales. Depuis plus d'un demi-siècle, les compositions des chanteurs de calypso sont présentes dans les espaces sociaux autres que ceux des sphères caribéennes d'immigrés. Dans ce contexte, transculturel et transnational, de circulation généralisée des compositions musicales, les chanteurs et (leurs) compositeurs s'accordent continuellement aux attentes renouvelées de leurs auditoires.

\section{Brèche dans la conversation}

Les sonorités musicales que certains établissements parisiens donnent à entendre en arrière-plan s'incrustent dans l'espace de ces lieux de rencontres et de discussion. Elles couvrent rarement les voix et autres bruits dont le volume ne manque pas de s'amplifier à mesure que les clients affluent. C'était le cas, ce jour de septembre 2013, dans ce café des Halles : une bande-son « jouait ». Elle était composée d'une série de morceaux de jazz et de blues de l'entre-deux-guerres et des années 1940. Soudain, inattendues, frappées de l'accent du Midwest américain, les voix des Andrews Sisters se faisaient entendre; la bande-son restituait leur interprétation de Rum and Coca-Cola dans son enregistrement initial de 1944.

J'en cite (au moyen d'une transcription) les deux premières strophes (lignes 1-4 et 10-13) et leur refrain (lignes 5-8, puis 14-17). Les lignes sont numérotées pour faciliter la présentation et le commentaire qui suivent ${ }^{7}$.

6. Trois films des années 1950 sont notoires : Affair in Trinidad (1952), pour quelques images de Trinidad qui situent les lieux de l'intrigue et son contexte colonial. Fire Down Below (1957) et Island in the Sun (1957), films tournés, pour le premier, à Trinidad, pour le second, à la Barbade et à Grenade. Parmi les acteurs de ces deux derniers films, on retrouve R. Mitchum (Fire down Below) et H. Belafonte (Island in the Sun). Belafonte $\mathrm{y}$ interprète, entre autres, deux chansons présentées comme des calypsos, dont celle qui donne son titre au film.

7. Selon un usage établi dans un article précédent (MASQUelier, 2011), chacune des transcriptions de cet article est présentée comme un texte traitant d'une situation, composée de multiples épisodes représentés dans les strophes et le refrain. Mais le texte transcrit n'est que cela : une transcription qui n'est qu'une mise en texte d'un énoncé (le résultat lui-même d'une mise en texte) indissociablement lié à une situation spécifique de production et d'écoute de parole. J'adjoins la lettre $T$ (pour transcription) à un nombre qui marque ici l'ordre de présentation (par exemple, $T 1$ ) : pour identifier chaque transcription, nécessairement incomplète, du document sonore, et ce qui y est « mis en texte » par les paroles chantées du calypso cité en référence ; que ces chansons aient été enregistrées en studio, ou lors d'une présentation publique (voir mon argumentation dans les paragraphes qui suivent). 


\section{Situation T1}

(1) If you ever go down Trinidad

(2) They make you feel so very glad

(3) Calypso sing and make up rhyme

(4) Guarantee you one real good time

(5) Drinkin' rum and Coca-Cola

(6) Go down point Koo Mah Nah

(7) Both mother and daughter

(8) Workin' for the Yankee dollar.

(9) Ob beat it man, beat it

(10) Since the Yankee come down to Trinidad

(11) They got the young girls all goin' mad

(12) Young girls say they treat'em nice

(13) Make Trinidad like paradise

(14) Drinkin' rum and Coca-Cola

(15) Go down point Koo Mah Nah

(16) Both mother and daughter

(17) Workin' for the Yankee dollar.

(18) Ob you vex me, you vex me

(19) etc.

Tel qu'il est transcrit, le texte reproduit l'orthographe qui est associée par copyright à la chanson des Andrews Sisters. Koo Mah Nab (ligne 6) s'y substitue à Cumana, le nom local de l'une des baies de la pointe nord-est de l'île de Trinidad. Cette orthographe de substitution, à n'en pas douter, sert de directive à la prononciation du nom trinidadien.

Le refrain est repris à l'identique, entre chaque strophe, tout au long de la chanson. Dans la version des Andrews Sisters, deux lignes de commentaires impromptus (lignes 9 et 18) sont, non pas chantées, mais parlées par l'une des trois interprètes de la chanson. Ces lignes simulent un aparté, mais il est difficile de déterminer quels destinataires sont interpellés. Elles font cependant partie intégrante de la chanson (c'est-à-dire de sa feuille de musique), si bien qu'elles sont répétées par d'autres interprètes de ce calypso dans leurs performances respectives, 
scéniques ou enregistrées ${ }^{8}$. Outre les deux lignes citées (ligne 9) Ob beat it man, beat it, (ligne 18) Ob you vex me, you vex me, un troisième commentaire, it's a fact, man, it's a fact ${ }^{9}$, s'insère entre le dernier refrain et les trois dernières lignes qui clôturent la chanson :

\section{Rum and Coca-Cola \\ Rum and Coca-Cola \\ Working for the Yankee dollar}

La chanson est entraînante. Elle participe de cet ensemble d'airs chantés par les Andrews Sisters, qui était régulièrement entendu sur les ondes des radios américaines pendant les années de guerre. Ces airs visaient à relever le moral des troupes ${ }^{10}$. Dans Rum and Coca-Cola, il est question de bon temps, de musique, de danse, de boisson, de plaisirs nocturnes. L'île est présentée comme un paradis (T1 lignes 1-4, ligne 13) : notamment, pour les jeunes Trinidadiennes (successivement nommées dans la chanson native girls et native peach) qui accompagnent les soldats de la garnison américaine en permission (T1 lignes 11-13). L'argent coule à flots.

Toutefois, les lignes du refrain comme celles qui terminent la chanson interrogent : figées par l'intervention technologique, reproduites en série, objet d'écoute passagère, d'attention flottante... que signifièrent ces lignes en 1946 pour leurs auditeurs du moment ? Que peuvent-elles signifier soixante-neuf ans plus tard dans les circonstances d'écoute évoquées précédemment ? Ces questions valent d'être posées; d'autant que l'on découvre que les interprètes elles-mêmes affirment être restées longtemps dans l'incertitude sur le sens d'une chanson enregistrée à la hâte, vers la fin d'une après-midi de travail en studio. Maxene Andrews, l'une des trois sœurs, l'a confié lors d'un entretien ${ }^{11}$ à un journaliste du New York

8. C'est le cas dans l'interprétation qu'en donne Arielle Dombasle, comme de la chanteuse trinidadienne Calypso Rose, lorsque l'une et l'autre interprètent la version des Andrews Sisters. 9. Traduction libre: allez décampe, mec, barre toi ; ça me chiffonne, tu m’agaces ; voilà c'est un fait, mec, c'est comme ça.

10. La popularité des chansons des Andrews Sisters, dans les années 1940, pourrait se mesurer à l'aune des millions de disques vendus. Mais un autre indice, plus indirect, semble tout autant significatif: en 1991, dix de leurs chansons les plus populaires, dont Rum and Coca-Cola, furent mises en scène sous la forme d'un ballet. Intitulé Company B (d'après l'une des chansons des Andrews Sisters), crée par Paul Taylor, présenté à Houston puis à New York, le ballet fut particulièrement bien accueilli par le public, et fit l'unanimité des critiques (voir Kisselgoff, New York Times, November 17, 1991).

11. New York Times, November 1, 1991. 
Times en 1991 : ce n'est que des années après l'enregistrement de Rum and Coca-Cola que leur fut révélé le « versant sombre » (pour traduire les mots de Maxene Andrews) de la phrase Both mother and daughter, working for the yankee dollar ${ }^{12}$. Innocence feinte ou bien réelle ? Que nous dit ce calypso?

Pour répondre à ces interrogations, cette étude fait appel à une notion clé de l'anthropologie linguistique, celle d'entextualization - notion proposée par Greg Urban et Michael Silverstein (1996). Je traduis entextualization par « mise en texte », pour souligner le caractère dynamique et processuel de la production de ce qui est signifié sous une forme particulière - un « texte »-dans le moment d'une énonciation (utterance) ou d'une performance. Le terme met en lumière le caractère situé de toute performance dans le moment de sa genèse, quelles qu'en soient les circonstances spécifiques (par exemple, une performance de paroles chantées sur une scène ou dans un studio d'enregistrement, etc.). Comment, de quelle manière, en quelle situation, un « texte » est-il « mis en texte »? La notion suggère que toute « mise en texte » s'inscrit dans une chaîne de décontextualisations et recontextualisations successives et que l'indexicalité du « texte $($ (« mise en texte ») est chaque fois relative à la situation du moment. La notion de « mise en texte » invite à ne pas penser le « texte » comme quelque chose de figé ou de statique, mais comme le résultat d'un procès de production dynamique qui révèle la valeur pragmatique de ce qui est signifié et généré (au moyen d'une pluralité de modalités sémiotiques), en situation (sous certaines circonstances), en contexte (par exemple, dans le cours d'une performance) ${ }^{13}$. Elle invite à appréhender le rapport dialectique entre « texte » et contexte en lien avec l'intertextualité dont le « texte mis en texte » participe (Masquelier, 2011). Enfin, je considère qu'une transcription est une « mise en texte ». Elle ne rend compte que de certains traits saillants. Elle n'est en aucun cas définitive ${ }^{14}$ et invite à prendre en compte le point de vue théorique qui la sous-tend (Ochs, 1979).

12. Traduction libre: Mère et fille, toutes deux, travaillent pour le dollar Yankee.

13. Dans la perspective de G. Urban et M. Silverstein, le « texte » ne peut en aucun cas être réduit à ce qui est « mis en mots».

14. Cette position est avant tout heuristique; elle s'accorde avec le dispositif que j'adopte pour les transcriptions qui sont présentées dans cet article. Il faut rappeler que les transcriptions indexent des situations d'énonciation, de performance et, en conséquence, de « mises en texte » particulières. Ces transcriptions participent ainsi de divers contextes de citation. Le procédé permet de repérer les variations d'un « texte » à un autre, des fragments stables (en quelque sorte) qui traversent les situations distinctes de « mises en texte $\gg$. 
CAHIERS DE LITTÉRATURE ORALE

202

Paroles publiques, paroles confidentielles $-n^{\circ} 77-78$

\section{Biographie d'un calypso : New York 1944-1947}

Faut-il s'étonner que le calypso des Andrews Sisters, enregistré par Decca en octobre 1944, puisse figurer dans la sélection d'un « paysage sonore » (soundscape) destiné à une écoute distraite de la part des clients d'une brasserie parisienne ? Ce choix concordait, en toute cohérence, avec l'ensemble thématique proposé : en l'occurrence, une sélection dédiée aux musiques et chansons des années 1940. D'ailleurs, dans cette période de Seconde Guerre mondiale, les trois interprètes de Rum and Coca-Cola participèrent, à plusieurs reprises, aux programmations récréatives (des services des armées américaines) destinées à distraire les troupes (Erb, 2009). Dans cette perspective, le libertinage frivole qu'esquisse la version de Rum and Coca-Cola chantée par les Andrews Sisters pouvait apaiser, et aider à renouer en imagination avec l'insouciance, ne serait-ce qu'un bref moment.

Toutefois, en s'invitant, impromptue, dans le cours de cette conversation parisienne de septembre 2013, la chanson des Andrews Sisters est devenue l'occasion d'une autre lecture : l'illustration du détournement d'un calypso et de ce qui en est fait quand il est recomposé hors de ses frontières locales pour un auditoire peu familier de cette forme de discours.

Dès ses premières ventes, à la fin de l'année 1944, le calypso des Andrews Sisters fut l'objet de publicité. Nous prendrons pour exemple ce qu'en dit le bref article que Time Magazine lui consacre le lundi 29 janvier 1945, jour de publication de l'hebdomadaire à cette époque, sous l'intitulé Music: Coca in Calypso ${ }^{15}$. L'article de Time Magazine, non signé (par un auteur en particulier), peut-être résultat d'une investigation collective de la part de l'hebdomadaire, une pratique alors habituelle, établit un certain nombre de faits. J'en fais ici une liste, tant ce court article est précis sur des points importants concernant ce calypso en particulier, et dont un lecteur attentif pouvait prendre note. 1) Il est reconnu que la chanson rencontre un succès certain, en raison du nombre des ventes du livret et du disque. 2) Il est noté que la chanson a été interdite d'antenne des stations radiophoniques majeures, et ce, pour trois raisons : pour publicité indirecte et non rémunérée de la marque de boisson Coca-Cola - où l'on apprend toutefois que l'entreprise avait tenté d'acquérir les droits sur la chanson, mais en vain ; pour la référence à l'alcool, l'invitation indirecte à en consommer ; pour le caractère licencieux (lustiness), voire libertin, de ses mots. 3) L'article précise que la chanson a été composée en 1943 par Rupert Grant, un chanteur trinidadien dont le nom de

15. Voir les archives de Time Magazine $:$ http://content.time.com/time/magazine/. 
scène est Lord Invador ${ }^{16}$. Il y est expliqué que ce calypso était chanté à l'origine par son auteur dans les lieux récréatifs ouverts aux militaires américains en garnison à Trinidad. L'hebdomadaire précise que la chanson, populaire à Port of Spain (principale ville administrative de l'île), était fredonnée par les soldats dans les cafés ou leurs casernes ${ }^{17}$.4) Time Magazine cite les sept premières lignes d'un texte - non pas celui chanté par les Andrews Sisters (voir plus haut, T1). Il est attribué à Rupert Grant ou Lord Invador.

\section{Situation T2}

(1) Since the Yankees came to Trinidad

(2) They have the young girls going mad

(3) Young girls say they treat them nice

(4) And they give them a better price

(5) They buy rum \& Coca-Cola

(6) Going down to Point Cumana

(7) Working for the Yankee dollars

La citation ne différencie ni strophe ni refrain. 5) L'hebdomadaire fait référence à un copyright déjà enregistré, à Trinidad, par l'un des services de l'administration coloniale britannique. 6) Une information vient compléter ces éléments sur l'historique de ce calypso en particulier : elle concerne la mélodie sur laquelle il est chanté ; selon l'hebdomadaire : une composition musicale dérivée d'une rengaine populaire à Trinidad.

Rum and Coca-Cola, dans sa version discographique, fut vendu au cours de l'année 1945 à plusieurs centaines de milliers d'exemplaires. Le succès commercial permit à la maison de disque, Decca, d'éviter la faillite. Toutefois, le calypso fut d'emblée l'objet d'une controverse sur des points dont se faisait déjà écho l'article du Time Magazine que nous avons cité plus haut. Elle prendra un tour judiciaire, en 1946 et 1947, avec deux procès pour cause de plagiat : un plagiat concernant le texte de la chanson, un autre sur la structure mélodique (Hill, 1993, p. 234-

16. Selon l'usage à Trinidad, chaque chanteur de calypso se faisait reconnaître sur scène par un sobriquet.

17. Dans les années 1940-1950, à Trinidad, les deux principaux sites militaires nordaméricains étaient situés dans la région de Chaguaramas, à une vingtaine de kilomètres à l'ouest de Port of Spain, et à Waller Field - un aéroport militaire jusqu'en 1949, situé à l'est de Port of Spain non loin d'Arima, un bourg des plaines du centre ; celles-ci s'étendent au pied des montagnes de la chaîne du littoral nord de l'île. 
CAHIERS DE LITTÉRATURE ORALE

204 Paroles publiques, paroles confidentielles $-n^{\circ} 77-78$

238 ; Neptune, 2007, p. 144-145). Cette publicité sera amplifiée en 1961, lorsque Louis Nizer, l'un des avocats de la défense ${ }^{18}$, publie ses mémoires. L. Nizer organise son ouvrage autour de quelques-unes de ses plaidoiries majeures afin d'illustrer son engagement juridique sa vie durant (Nizer, 1961). Il en vient à consacrer cinquante-trois pages au procès pour plagiat (Nizer, 1961, p. 233-286). Ce cas, depuis qu'il a été porté à l'attention des juristes nord-américains, est désormais exemplaire : un résumé de la procédure ${ }^{19}$ et le jugement, tel qu'il fut initialement énoncé puis confirmé en appel, sont accessibles sur le site de documentation de la faculté de droit (graduate school) de l'université de Columbia dont L. Nizer était diplômé. Les plaignants - Lord Invador comme auteur de la composition originale, Lionel Belasco en tant que compositeur de la mélodie - eurent gain de cause ; des compensations financières leur furent versées. Les droits commerciaux sur la version chantée par les Andrews Sisters ne leur furent pas restitués pour autant, sans doute, à l'issue d'un arrangement entre les parties opposées.

Il faut noter que lors du second procès, à propos des droits sur la composition mélodique, il fallut débattre de la question de savoir si la composition dite « originale » de Belasco n'était pas, de fait, la transposition ${ }^{20}$ d'une vieille rengaine populaire d'origine martiniquaise intitulée l'Année passée, une chanson connue à Trinidad à la fin du XIX ${ }^{\mathrm{e}}$ siècle, comme dans les années 1950, selon les interlocuteurs d'Emory Cook ${ }^{21}$. Le premier procès permit d'établir sans nul doute l'originalité de la composition chantée par Lord Invador en 1943. Elle avait été publiée par le biais d'un recueil de calypsos chantés au Calypso Victory Tent dans les premiers mois de 1943. Le calypso avait été enregistré par ailleurs par le gérant du lieu (Mohammed Khan) auprès des autorités coloniales britanniques de Trinidad. Une partie de ce document fut soumis comme preuve, en soutien de la déposition de Lord Invador. Nous en proposons une brève analyse plus loin.

18. Spécifiquement, dans le procès qui portait sur la mélodie de Rum and Coca-Cola composée par Lionel Belasco, un Trinidadien.

19. Le document, accessible sur http://mcir.usc.edu/, est identifié sous la référence : Baron v. Leo Feist 78 F. Supp. 686 (S.D.N.Y. 1948).

20. Lionel Belasco était né en 1882 à la Barbade, au sein d'une famille créole de musiciens. Pianiste, il s'est intéressé aux formes musicales populaires dès le début du $\mathrm{Xx}^{\mathrm{e}}$ siècle. Il s'est fait connaître comme interprète et compositeur, mais aussi pour ses transcriptions de nombreux airs du répertoire musical populaire des Caraïbes (voir discographie).

21. Dans les premières décennies du $\mathrm{Xx}^{\mathrm{e}}$ siècle, il était encore courant pour les auteurs de calypso de les chanter sur des mélodies populaires connues du public trinidadien. Selon Holis Liverpool (2001), une dizaine de ces mélodies constituaient ainsi un répertoire mélodique commun. 


\section{Situation T3}

Cour de justice: text of Rum and Coca-Cola as published by KhanRum and Coca-Cola (by Invador)

(1) Since the Yankees came to Trinidad

(2) They have the young girls going mad

(3) The young girls say they treat them nice

(4) They buy rum and Coca-Cola

(5) Go down Point Cumana

(6) Both mother and daughter

(7) Working for the Yankee dollar

(8) I had a little mopsy the other day

(9) Her mother came and took her away

(10) Then her mother and her sisters

(11) Went in a car with some soldiers

(12) They buy rum and Coca-Cola, etc.

(13) There are some aristos in Port of Spain

(14) I know them well, but I won't call names

(15) In the day they wouldn't give you a right

(16) But you can see them with the foreigners

(17) Late at night

(18) A couple got married one afternoon

(19) And was to go to Mayaro on a honeymoon

(20) The very night the wife went with a Yankee lad

(21) And the stupid husband went staring mad

(22) Inspector Jory did a good job

(23) At St. James he raid a recreation club

(24) There was carrying on the club as a brothel

(25) The condition in which he found the girls

(27) I cannot tell 
CAHIERS DE LITTÉRATURE ORALE

\section{Performances scéniques : New York, 1946, Port of Spain, 1950}

Comme je l'ai suggéré auparavant, le milieu des années 1930 marque un tournant pour la reconnaissance du calypso, en particulier à New York. Plusieurs chanteurs et musiciens trinidadiens y auront contribué ${ }^{22}$. Certains d'entre eux étaient émigrés et résidents. Ils tentaient de faire profession de leur art. D’autres étaient invités ponctuellement pour y réaliser des émissions de radio ou participer à des concerts. Ils prolongeaient leur séjour grâce à des engagements sur les scènes de clubs, notamment au Village Vanguard, dans le quartier de Greenwich, au sud de Manhattan (Eldridge, 2002, p. 621-623 ; Hill, 1993, p. 159-166). La presse écrite - dont Time, Newsweek, The New Yorker, The New York Times, Life, Billboard - ne manquait pas de relayer cet intérêt pour le calypso.

La présence de Lord Invador à New York, à partir des premiers mois de l'année 1945, avait comme raison le procès en plagiat qu'il avait décidé d'intenter pour faire reconnaitre ses droits de compositeur de la version originale de Rum and Coca-Cola, chantée à Trinidad, en 1943, dans les clubs ouverts à la garnison américaine présente sur l'île depuis l'été 1941. Habitué de New York, Lord Invador y résidera ${ }^{23}$, entre allers et retours depuis Port of Spain, séjours en Grande-Bretagne et autres voyages. L'un des épisodes majeurs de ses séjours new-yorkais aura été sans doute sa participation, le 21 décembre 1946, au concert organisé par l'association People's Songs au Town Hall Theater, situé dans le centre de Manhattan ${ }^{24}$. Il

22. Parmi les chanteurs invités de cette période, deux étaient très connus à Trinidad sous leurs sobriquets : Atilla $(s i c)$ the Hun, et The Lion. Wilmoth Houdini, Lord MacBeth the Great, The Duke of Iron, quant à eux, étaient émigrés résidents à New York depuis la fin des années 1920 ou le début des années 1930. Tous ces chanteurs de calypso enregistraient sous leur nom de scène. Ce n'est que dans les années 1980 que l'usage du sobriquet s'est relâché.

23. Au cours de ses années de résidence new-yorkaise, Lord Invador a enregistré pour les compagnies de Moses Asch (successivement, Disc Records et Folkways). Ces entreprises étaient dédiées aux musiques populaires (folk music). Reconnu pour ses compositions de calypso, Lord Invador avait le mérite de maîtriser par ailleurs un large éventail de styles (de chansons) qui constituait un excellent échantillon du répertoire musical populaire trinidadien du milieu du xx ${ }^{\mathrm{e}}$ siècle. En 2000, Smithsonian Folkways Recordings a publié une sélection des enregistrements de Lord Invador, enregistrements réalisés à l'initiative de Moses Asch, et dont certains n'avaient pas été commercialisés. Cette collection révèle l'étendue du répertoire musical de Lord Invador (voir discographie). Lord Invador est mort à New York en octobre 1961.

24. Le succès du concert au Town Hall en décembre 1946 s'est traduit pour Lord Invador par au moins deux engagements au Carnegie Hall de New York dans l'année 1947. 
s'agissait en fait d'une présentation des traditions musicales de Trinidad, dont faisaient partie les différents styles de calypso. L'ethnomusicologue Alan Lomax en était le «maître de cérémonie » et c'est à son initiative que l'ensemble $\mathrm{du}$ concert fut enregistré. Longtemps oubliés au fond d'une armoire, ces enregistrements ont fait l'objet d'une publication en 1999 par Rounder Records, maison d'édition discographique qui diffuse aujourd'hui les archives sonores d'Alan Lomax. Annotée par les anthropologues et musicologues John Cowley et Donald Hill, cette publication est accompagnée d'une transcription complète des paroles énoncées et des calypsos chantés, ce soir-là, par deux spécialistes du créole anglophone, Ronald et Willy Kephart ${ }^{25}$. C'est sur cette documentation sonore et des transcriptions revisitées par mes soins que j'appuie l'ensemble des remarques qui suivent.

L'ensemble du concert du Town Hall restitue, d'une certaine manière, le format de présentation des performances des chanteurs de calypso dans les lieux (calypso tents) qui leur étaient familiers à Trinidad. Un spectacle habituel de calypso est assuré en effet par un groupe de chanteurs. Au Town Hall, il s'agissait de Lord Invador, MacBeth the Great, ainsi que the Duke of Iron. Chanson après chanson, chacun se produit sur scène tour à tour, introduit à chaque fois par un « maître de cérémonie », rôle joué par Alan Lomax lors du concert du Town Hall. Or, en cette soirée du 21 décembre 1946, le premier calypso, qu'Alan Lomax invite Lord Invador à chanter, n'est autre que Rum and Coca-Cola. En introduisant Lord Invador, Lomax lui donne l'occasion de définir ce qu'est un calypso et d'expliquer la raison de sa composition. Après avoir présenté le calypso trinidadien comme une forme de poésie chantée (a style of poetry [...] in song) qui porte sur des événements d'actualité, Lord Invador rend compte des faits qui l'avaient inspiré en 1943 : les soldats américains avaient l'habitude de distribuer des bonbons, de faire de petits cadeaux. Les opportunités de rencontres avec les jeunes Trinidadiennes étaient fréquentes. Ces soldats coupaient leur rhum avec du Coca-Cola. À écouter Lord Invador, il ne s'agit pas de détails anodins, mais des conséquences de ce qu'il nomme dans son commentaire : l'invasion sociale américaine (American social invasion). Les rires de l'auditoire, en réponse à cette description des faits, suggèrent que le non-dit ne lui avait pas échappé, que ces formulations étaient entendues comme métonymiques de situations sans doute plus sombres. Il est probable que cet auditoire-là était en grande partie au courant

25. Voir discographie : «Calypso at Midnight » et « Calypso after Midnight », deux CD publiés par Rounder Records en 1999. 
du litige judiciaire qui opposait Lord Invador à ses plagiaires. À l'issue de cette introduction, celui-ci chantera sa version de Rum and Coca-Cola.

La présentation du calypso sur la scène du Town Hall respecte quelques-unes des habitudes trinidadiennes qui gouvernaient toute performance scénique d'un calypso à cette époque. Outre l'annonce du calypso et de son auteur/chanteur par le maître de scène ou de cérémonie, la chanson est introduite par une partie musicale orchestrale ; ici un orchestre réuni par le musicien caribéen Gerald Clark (guitare à six cordes, violon, batterie, hochets appelés chac-chac, clarinette, basse, piano). Mais lors de la performance des strophes et du refrain par le chanteur, l'orchestre s'efface, à l'exception du jeu d'instruments (chac-chac, basse, batterie). C'est en arrière-plan que l'orchestre accompagne le chanteur. Les paroles des strophes sont ainsi mises en avant. Les refrains sont repris en chœur, ici (sur la scène du Town Hall) par l'ensemble des chanteurs présents et par certains des musiciens qui mêlent leurs voix. Dans cette performance publique de Rum and Coca-Cola, le refrain est chanté une seconde fois mais sans la participation de Lord Invador, suivi par un insert orchestral qui précède la reprise du chant à la strophe suivante. L'alternance entre chanteur principal et chœur (pour l'exécution du refrain) comme entre chanteurs et musiciens restitue la forme appel-réponse (call-andresponse) des formes musicales qui, dans la première moitié $\mathrm{du} \mathrm{xx}^{\mathrm{e}}$ siècle, ont influencé le calypso de Trinidad ${ }^{26}$.

\section{Situation T4}

(1) And when the Yankees first went to Trinidad

(2) Some of the young girls were more than glad

(3) They said that the Yankees treat them nice

(4) And they give them a better price

(5) They buy rum and coco cola

(6) Went down point Cumana

(7) Both mothers and daughters

26. Le terme d'énonciation, celui qui est associé à l'approche énonciative du langage chez Émile Benveniste, pointe vers les marques de subjectivité énonciative de l'énoncé. Sans exclure cette perspective, notre approche met l'accent sur la performance de l'interlocution, l'acte par lequel la dimension pragmatique d'un dire prend corps et se réalise dans une relation interpersonnelle. Cette perspective est plus apte à mettre en lumière l'événement singulier de toute performance enregistrée en public (live). Certaines des transcriptions restituent les marques de cette activité interlocutive (MASQUELIER, 2011, 2012). 
(8) Working for the Yankee dollar

(9) Ah, look, I had a little chick the other day

(10) But her mother came and took her away

(11) Herself, her mother and her sisters

(12) Went in a cab with some soldiers

(13) They bought rum and coco cola

(14) Went down point Cumana

(15) Both mothers and daughters

(16) Working for the Yankee dollar

(17) They have some aristos in Port of Spain

(18) I know a lot but I wouldn't call name

(19) And in the day, they wouldn't give you a right

(20) But you could see them with the foreigners late at night

(21) Drinking

(22) Rum and coco cola

(23) Going down point Cumana

(24) Both mothers and daughters

(25) Working for the Yankee dollar

(26) I know a couple who got married one afternoon

(27) And was to go to Miami on their honeymoon

(28) But the bride run away with a soldier lad

(29) And the stupid husband went staring mad

(30) They bought rum and coco cola

(31) Going down point Cumana

(32) Both mothers and daughters

(33) Working for the Yankee dollar

Une comparaison des transcriptions $T 3$ et $T 4$ - respectivement celle du texte présenté comme preuve d'une composition originale de ce calypso par Lord Invador (au cours du procès de 1946) et celle du texte chanté sur scène lors du concert du Town Hall - révèle que le chanteur, lors du concert du Town Hall, reste fidèle aux épisodes qui constituent le contenu des strophes de T3. Chacune des strophes de $T 3$ et $T 4$ scénarise des épisodes (événements ou observations). 
Ces épisodes décrivent : la joie des jeunes Trinidadiennes à faire affaire avec les soldats de la garnison américaine qui consentent à mieux payer (T4lignes 1-4); la manière dont le chanteur a perdu son amie à l'issue de l'intervention de la mère de cette dernière, parce que mère et sœurs s'en vont partager avec les soldats un même transport (T4 lignes 9-12) ; les connivences nocturnes entre étrangers et gens de bonne famille locale (Aristos in Port of Spain), ceux-là qui ne serrent aucune main au grand jour (T4 lignes 17-20) ; la rupture d'un mariage célébré un aprèsmidi, à la veille d'un voyage de noces, parce que la mariée a décidé de rejoindre un soldat (T4 lignes 26-29). Chaque strophe est ponctuée d'un même refrain : l'alliance du rhum et du Coca, sur les plages de Cumana, suggère l'esprit festif de ces complicités. La conclusion est plus énigmatique : mères et filles travaillent pour le dollar yankee (T4 lignes 7-8, 15-16, etc.).

Lors de sa performance au Town Hall, Lord Invador a opéré quelques modifications de vocabulaire pour ajuster ses expressions à son auditoire new-yorkais : il substitue little chick (T4 ligne 9), une expression qui fait référence de manière familière à une « fille », à little mopsy (T3 ligne 8). Cette dernière expression était courante dans le parler de Trinidad des années 1940 ; elle désignait une compagne amoureuse. Plus important, il omet de chanter la dernière strophe de sa composition initiale de 1943 (T3 lignes 22-27) : elles décrivaient la prostitution clandestine de certains clubs du quartier de St. James, un faubourg de Port of Spain.

Au cours de la dernière heure du concert, l'auditoire du Town Hall eut l'opportunité d'entendre deux calypsos qui complétaient les propos de Lord Invador sur les conséquences de la présence militaire américaine à Trinidad durant ces années de guerre. Intitulé The GI and the Lady, le premier de ces calypsos est une saynète chantée à trois. Cette forme de performance est appelée calypso drama ; elle fut imaginée dans les années 1940 par les chanteurs de calypso pour renouveler le genre (Hill, 1972). Le calypso drama présenté au Town Hall de New York est chanté par Lord Invador, dans le rôle d'un chanteur de calypso sur le point d'épouser une jeune fille. Cette dernière est jouée par Macbeth the Great. Dans ce petit drame, la jeune femme est séduite par un soldat américain, incarné par The Duke of Iron. Ce calypso à trois voix (masculines) est organisé comme une brève tragi-comédie musicale : selon les événements qui y sont rapportés, parce que sa fiancée porte de l'intérêt à un soldat étranger, le fiancé (chanteur de calypso) l'a battue avec violence. Il tente de s'excuser de son geste. Il implore la jeune femme de lui renouveler son amour, lui rappelle que le mariage est sur le point d'être scellé. La jeune femme rompt la relation et déclare qu'elle rejoint le soldat. Ce dernier, dit-elle, souhaite l'épouser et lui offrir une vie d'abondance à Brooklyn : voiture de luxe, argent, robes, appartement. En comparaison, la jeune femme 
établit la liste des imperfections de son fiancé trinidadien. Elle prend note de son insolvabilité et lui annonce : I am going to the States with the Yankee soldier (je pars aux États-Unis avec le soldat yankee). Le second calypso est intitulé Yankee dollar. Il précède la chanson de clôture du concert. Enregistrée à New York par Lord Invador dans le courant de l'année 1946, chantée sur une mélodie populaire connue à Trinidad, cette composition du chanteur réitère combien séduisante était la richesse des soldats nord-américains. Le thème, coutumier désormais, est celui du fiancé trinidadien trompé par celle qui lui préfère un soldat argenté. Non sans humour, le refrain fait référence au chanteur et compositeur de Rum and Coca-Cola : la notoriété acquise pour avoir chanté ce calypso ne peut supplanter le pouvoir d'attraction du Yankee dollar (T5 lignes 9-12).

\section{Situation T5}

$[\ldots]^{27}$

(7) So, she told me

(8) She love Yankee money

(9) And she said Lord Invador

(10) Not because you sung rum and coco cola

(11) Don't bother

(12) If you know you ain't got that Yankee dollar

\section{$[\ldots]$}

Le calypso se termine toutefois sur une note d'espoir : la fin de la guerre annonce le départ des troupes, et le retour à des temps plus favorables aux soupirants trinidadiens, comme aux valeurs plus modestes du shilling britannique.

À son retour à Trinidad, Lord Invador n'a pas cessé de présenter sa version de Rum and Coca-Cola. Deux enregistrements ${ }^{28}$ de 1950, réalisés en studio et en public (live), révèlent qu'il avait augmenté sa composition initiale d'une strophe,

27. Le signe [...] indique les passages qui sont omis par la transcription : soit les paires de strophe/refrain qui précèdent, soit celles qui font suite au texte transcrit.

28. L'enregistrement public est attribué à John Bessor par Donald Hill (voir discographie et Hill, 1993, p. 234-240). L'enregistrement selon Hill aurait eu lieu dans le club (calypso tent) dont Lord Invador était devenu le propriétaire. Ma transcription est établie sur la base de cet enregistrement. La version de studio a été publiée dans la collection Trinidad Calypso 1939-1959 de Frémeaux \& Associés. 
CAHIERS DE LITTÉRATURE ORALE

212 Paroles publiques, paroles confidentielles $-\mathrm{n}^{\circ}$ 77-78

dans sa version scénique chantée par exemple lors du concert au Town Hall de New York. Elle vient clôturer la version scénarisée du calypso ${ }^{29}$ :

\section{Situation T6}

$[\ldots]$

(29) Singing

(30) Rum and coco cola

(31) Go down point Cumana

(32) Both mothers and daughters

(33) Working for the Yankee dollar

(34) I wonder what is your interested opinion

(35) We haven't got no bad speaking Trinidadian

(36) We never say "caca cola"

(37) Neither did we say Yankee dallah

[...] refrain, etc.

Ce commentaire « métalinguistique » à propos de l'énonciation des mots Coca-Cola et dollar (lignes 34-37) ne manque pas d'ironie : il affirme de surcroît, dans le même mouvement, le droit et la fierté à parler différemment (ligne 35), avec l'économie des moyens linguistiques qui caractérise les compositions de calypso. En faisant porter la controverse sur deux mots emblématiques, c'est métonymiquement que Lord Invador distingue la variante de l'anglais parlé à Trinidad. Coca-Cola en anglais de Trinidad se prononce coco cola et dollar n'est pas entendu comme dallah. C'est aussi une manière pour Lord Invador de faire entendre l'originalité phonique (et irréductible) de sa composition, de la distinguer de la copie new-yorkaise qu'en donnent les Andrews Sisters.

29. L'enregistrement public de John Bessor est un document particulièrement significatif pour l'étude du paysage sonore des performances scéniques, comme de leur accomplissement. Premièrement, la performance du calypso par Lord Invador est accomplie dans un contexte sonore d'arrière-plan que le terme de «brouhaha » pourrait décrire. Deuxièmement, le refrain du calypso est repris par le public de bout en bout de la performance. La voix de Lord Invador s'en trouve presque couverte à chaque fois. Enfin, la performance du calypso est ponctuée par deux improvisations du chanteur. Elles lui permettent de s'adresser à l'un des membres de l'auditoire et ceux qui l'accompagnent, en postulant ce qui pourrait bien être leur évaluation du calypso chanté : «Ifyou do appreciate this calypso/ you have some people there in your company/I sure they appreciate the melody/... It seem as you love this calypso ». 


\section{Dire et écrire les violences de l'histoire}

Je réitère un argument énoncé dans une autre étude (Masquelier, 2011) : les paroles d'un calypso, celles qui sont composées chaque année et chantées pour et avec un auditoire durant la période du carnaval de Trinidad, ne sont pas éphémères. Bien qu'elles soient celles d'une saison ou celles du moment d'une performance singulière, dans la perspective des participants aux fêtes ou de la sphère publique des résidents nationaux de l'île, ces chansons, pour nombre d'entre elles du moins, participent au fil du temps d'un espace de références et de textes. Ceci est vrai quel que soit le style sous lequel ces paroles se présentent, sous la forme de strophes improvisées ou scénarisées : comme chanson grivoise, commentaire politique d'un événement du présent, conte moral. De fait, de nombreux relais génèrent cet espace de référence et de médiations. Cet espace se présente sous différentes formes de modalités et de matérialités. Les conversations (quelles que soient leurs circonstances et raisons) contribuent à le constituer, tout comme y participent les médias, l'industrie du disque, les recherches à caractère ethnomusicologique, les travaux universitaires. Cet espace n'est donc pas seulement textuel, au sens littéral ; il est aussi fait de sons, d'images, de situations, notamment celles de performances singulières, spontanées, parfois rejouées à l'occasion. L'énonciation d'un calypso ne peut se réduire au seul texte chanté à l'occasion d'une performance scénique ni au seul texte chanté-enregistré que l'on peut réentendre à l'occasion. Un calypso est aussi un texte susceptible d'être cité, en conversation, comme dans une œuvre de fiction. C'est ce dernier point que je voudrais explorer.

Deux fictions, écrites dans les années 1950 par des auteurs trinidadiens de la diaspora, dont les renommées ne sont pas analogues, contextualisent les commentaires mis en chansons par Lord Invador. Publié tardivement en 1959, à Londres, Miguel Street est l'un de ces deux textes littéraires. L'ouvrage est de fait le premier roman écrit par Vidia Naipaul (né en 1932) dans ses premières années passées en Grande-Bretagne ${ }^{30}$. Le second, intitulé Rum and Coca-Cola, publié à Melbourne en 1956, est écrit par Ralph de Boissière (1907-2008). Ce dernier est né à Trinidad comme V. Naipaul. De classes sociales distinctes (dans le contexte trinidadien), leurs trajectoires biographiques sont singulières. L'un (V. Naipaul)

30. Lauréat du prix Nobel de littérature en 2001, V. Naipaul, est désormais citoyen britannique et réside à Londres. V. Naipaul a effectué une brève visite à Trinidad en avril 2007. Présent à Trinidad à cette période, j'ai pu assister à la lecture publique, le 20 avril 2007, que V. Naipaul a faite sur le campus de l'université des West Indies (à St. Augustine, Trinidad) de quelques extraits de son œuvre, en particulier de deux des chapitres de Miguel Street. 
est Indo-Trinidadien, fils d'un journaliste du Trinidad Guardian, issu d'une famille brahmane arrivée à Trinidad avec les migrations de main-d'œuvre (indentured labor) que la puissance coloniale britannique a organisées depuis l'Inde, entre le milieu du XIX ${ }^{\mathrm{e}}$ siècle et la Première Guerre mondiale. L'autre (R. de Boissière) est d'une famille créole, d'origine française et de descendance africaine par sa grand-mère paternelle. Tous deux furent éduqués au Queen's Royal College, l'une des meilleures écoles secondaires de Port of Spain. Ils feront chacun le choix de l'émigration : V. Naipaul, en 1950, vers la Grande-Bretagne, grâce à l'obtention d'une bourse qui lui permettait de poursuivre ses études universitaires à Oxford. R. de Boissière, en 1947, vers les États-Unis pour un bref séjour de quelques mois avant de poursuivre son émigration définitive vers l'Australie. Les pages qui suivent esquissent quelques-uns des liens que tissent Miguel Street et Rum and Coca-Cola avec les compositions de Lord Invador.

Le narrateur de Miguel Street est un jeune garçon anonyme. Depuis les marches de sa résidence, jusqu'à son départ de Miguel Street - départ qui clôture le récit il tient une chronique fragmentée ${ }^{31}$ de ce microcosme social : une scène animée, à l'échelle d'une rue d'un faubourg de Port of Spain, par les destins de gens de vie modeste, et les péripéties de leur quotidien.

Un étranger qui traversait en voiture Miguel Street dirait « pouillerie », parce qu'il ne verrait rien d'autre. Mais nous qui y vivions, voyions notre rue comme un monde où chacun était très différent de l'autre. Man-Man était fou ; George stupide ; Big Foot un matamore ; Hat un aventurier ; Popo un philosophe ; et Morgan notre comédien ${ }^{32}$.

Le cadre temporel d'arrière-plan est fixé par le souvenir d'événements qui eurent lieu dans les années 1930 et par les changements sociétaux que la présence militaire américaine à Trinidad aura générés à partir de 1941. Dans le récit de V. Naipaul, Miguel Street est présenté comme une rue parmi d'autres où se manifestent, à l'échelle des biographies individuelles des membres de cette communauté de voisinage, les conséquences visibles des transformations qui emportent Trinidad. Simultanément, elle se présente comme l'une des coulisses où les différents personnages prennent acte de ce monde inattendu, imposé de

31. La rue (Miguel street) donne au récit de V. Naipaul son unité de lieu. La série des chapitres fait traverser le lecteur dans le temps : il est fait d'épisodes disjoints (chapitres) qui se font écho au cours de plusieurs années.

32. Vidia S. Naipaul, 1967 , p. 84. 
l'extérieur, pour se préparer à y opérer à l'échelle de la ville et de l'île. Je cite V. Naipaul :

À cette époque, les Américains grouillaient à Port of Spain, donnant à la ville une atmosphère du tonnerre. Les enfants n'avaient pas mis longtemps à s'apercevoir que c'étaient des braves types, toujours prêts à donner des deux mains. Hat eut vite fait de monter une petite combine. Il avait chargé cinq d'entre nous de mendier dans toute la ville du chewing-gum et du chocolat. Pour chaque paquet de chewing-gum que nous lui remettions, il nous donnait un $c e n t^{33}$.

Composé de dix-sept courts chapitres ${ }^{34}$, le récit restitue les fils qui lient les différents personnages, résidents, passagers ou permanents, de ce voisinage. L'une des clés de lecture est donnée dès le premier chapitre qui introduit le personnage de Bogart (nommé - c'est explicitement énoncé par le narrateur - d'après l'acteur du même nom) : tailleur de profession, sans clients, il va et vient, s'absentant parfois à l'improviste pour de longs mois, à la poursuite d'occupations quelque peu énigmatiques, peut-être illégales. Toutefois, fait remarquable, à chacun de ses retours à Miguel Street, l'anglais américain de Bogart s'est amélioré et ses attitudes se modèlent toujours mieux à celles du Bogart du film Casablanca.

Lorsqu'il revint, il avait un peu grossi, mais il était devenu un peu plus agressif et son accent était à présent typiquement américain. Pour compléter l'imitation, il commença à se montrer expansif avec les enfants. Il les appelait et leur distribuait de l'argent pour acheter du chewing-gum et du chocolat. Il aimait leur tapoter la tête et leur donner de bons conseils. La troisième fois qu'il partit et qu'il revint, il réunit dans sa chambre tous les enfants [...]. Il acheta des caisses de Solo, de Coca-Cola et de Pepsi-Cola, et des tas de gâteaux ${ }^{35}$.

33. Vidia S. Naipaul, 1967, p. 74.

34. Chacun des courts chapitres porte un titre; deux d'entre eux sont empruntés à des calypsos : Love, Love, Love, Alone (L'amour, l'amour, l'amour seul) est une citation d'une ligne du refrain d'un calypso enregistré par Lord Caresser, en 1937, commentant l'abdication, en 1936, d'Edward viII, afin d'épouser une roturière américaine divorcée. Le second titre, Until the Soldiers Came (Avant l'arrivée des soldats), fait référence à un calypso de Lord Invador, enregistré en mai 1945 à New York : The soldiers Came and Broke up my Life; ce calypso en préfigure un autre, Yankee dollar, enregistré la même année et chanté au Town Hall en décembre 1946 (voir discographie).

35. Vidia S. Naipaul, 1967, p. 16. 
Dans ce petit monde, les différents protagonistes, au fil des intrigues qui les associent, citent des extraits de calypsos : pour ponctuer un commentaire, illustrer une situation, mettre un nom ou un titre sur un événement. En cela, le roman de V. Naipaul prend acte du rôle public que jouent les calypsos dans la vie quotidienne à Trinidad. Au chapitre quinze, le narrateur rapporte l'anecdote d'une sortie nocturne, rocambolesque, insouciante, organisée par Edward, frère de Hat (l'un des personnages emblématiques de Miguel Street), sous prétexte d'aller pêcher le crabe. Mais le chapitre bascule abruptement sur le récit de l'irrémédiable transformation d'Edward à l'issue de l'arrivée américaine à Trinidad. Je cite les lignes qui marquent la frontière entre l'insouciance et la drôlerie du récit d'une pêche aux crabes qui n'eut jamais lieu - faute de crabes - et le compte rendu des transformations dramatiques qu' incarne Edward dès le moment où il s'associe aux Américains ${ }^{36}$.

Alors, la guerre éclata. Hitler envahit la France et les Américains envahirent la Trinidad. Lord Invador remporta un succès avec son calypso :

Je vivais avec ma ferme honnête et heureuselavant que les soldats viennent et brisent ma vie

Il y eut pour la première fois du travail pour tout le monde à la Trinidad. Invador chanta :

Père, mère et scur/travaillent pour le dollar américain/De l'argent dans le pays !/Le dollar américain, ob!

Edward [...] alla travailler chez les Américains à Chaguaramas [...]. Edward se laissa complètement grignoter par les Américains. Il commença par s'habiller comme eux, à mastiquer du chewinggum et il essaya de parler avec l'accent américain. Nous ne le vîmes plus beaucoup, sauf le samedi, et alors, nous nous sentions petits et inférieurs à côté de lui [...]. À entendre parler Edward, nous sentions que l'Amérique était un pays gigantesque habité par des géants vivant dans des maisons énormes et roulant dans les plus grosses voitures du monde ${ }^{37}$.

36. Il faut noter que dans l'extrait cité les énoncés en italiques (dans la version française du roman de V. Naipaul) sont empruntés aux calypsos de Lord Invador.

37. Vidia S. Naipaul, 1967, p. 200-201. 
Le roman de R. de Boissière Rum and Coca-Cola fait suite à une première fiction Crown Jewel publiée en 1952 en Australie ${ }^{38}$. Le ton des deux récits s'accorde avec les engagements politiques de gauche de l'auteur et le souhait d'écrire des fictions réalistes et historiques. R. de Boissière disait aussi admirer la littérature russe du $\mathrm{XIX}^{\mathrm{e}}$ siècle. Crown Jewel et Rum and Coca-Cola forment un tout. Ils portent sur deux décennies de transformations sociales à Trinidad. Le premier récit (Crown Jewel) traite des grandes grèves qui secouèrent Trinidad en 1937, dans le milieu agricole et l'industrie pétrolière. Ces événements s'inscrivaient dans la continuité des difficultés économiques et des tensions politiques que connurent les Caraïbes anglophones dans leur ensemble à partir du début des années 1930.

Rum and Coca-Cola a pour objet les bouleversements des années 1940. Cette fiction propose une fresque minutieuse du changement social engendré par la présence de la garnison américaine à Trinidad. Les conséquences de cette présence furent politiques, économiques, sociales et culturelles. Elles se réfractèrent dans l'ensemble de la « société » coloniale trinidadienne : au sein de la bourgeoisie créole d'origine européenne comme dans la classe populaire, qu'elle soit de descendance indo-trinidadienne ou d'origine africaine. L'ouvrage de R. de Boissière s'attache à décrire les multiples facettes de cette situation, au moyen d'anecdotes, de commentaires, d'intrigues. Les causes matérielles du changement sont présentées sans détour : les travaux d'infrastructures engagés par la présence nord-américaine ainsi que le besoin de services divers ouvrirent un grand nombre d'emplois peu qualifiés. De nombreux Trinidadiens saisirent ces opportunités. Les salaires payés par l'employeur américain, en général plus élevés que sous le régime colonial britannique, et l'afflux d'argent mis en circulation ont bouleversé les équilibres des transactions du quotidien. R. de Boissière montre les effets de cette économie sur le régime colonial en place. Il décrit comment l'ordre colonial britannique de l'époque s'est fissuré, comment le pouvoir économique de l'élite créole a vacillé.

Mais l'auteur dépeint aussi de façon acerbe et ironique les manières d'être américaines et les valeurs de ce nouveau monde qui s'imposaient avec force et que de nombreux Trinidadiens cherchaient à copier ou à maîtriser à leur avantage, parfois pour y survivre au mieux. Les comportements (sociaux et culturels) des militaires,

38. R. de Boissière compte parmi les fondateurs dans les années 1950 de l'Australian Book Society, une coopérative soutenue partiellement par le mouvement ouvrier australien. Les deux romans cités dans cette étude furent publiés initialement par cette coopérative. Rum and Coca-Cola, initialement publié en 1956, a fait l'objet de révisions substantielles pour la seconde édition de 1984. Cette seconde édition est celle qui est commentée dans ces pages (voir bibliographie). 
tout comme les préjugés discriminatoires véhiculés au sein de cette armée, étaient scrutés dans le moindre détail. Les chanteurs de calypso se firent les porte-parole de ces observations et réflexions populaires. Le titre de l'ouvrage est une référence explicite au calypso de Lord Invador. L'un des personnages principaux du roman, Fred Collingwood, est un syndicaliste engagé dans la contestation des pouvoirs établis, mais il est aussi compositeur-chanteur de calypso, à ses heures de loisir.

\section{Conclusion}

Nous conclurons la biographie sociale (juridique et politique) de Rum and Coca-Cola, telle qu'elle est proposée en arrière-plan de nos divers commentaires sur la poétique du calypso, par deux remarques. En premier lieu, il faut rappeler ce que nous avons tenté de montrer. L'ensemble des formes « textuelles » prises en compte participe d'une même trame : une toile constituée de fragments, de résidus, de morceaux (de discours), de mots cités ${ }^{39}$. Ils se font écho, se répondent les uns aux autres. Cette trame dialogique est tout à la fois interne (à une même chanson) et externe (entre les chansons).

La forme de l'appel-réponse (call-and-response) est interne au calypso sous la forme de l'alternance entre strophe et refrain, mais aussi sous la forme de commentaires énoncés (chantés-parlés) sur scène par les chanteurs dans le cours de la performance comme dans certains des enregistrements de studio. En outre, tout calypso, dans la conception trinidadienne de ce genre de parler-chanter, est susceptible de répondre à d'autres chansons, ou de solliciter une réponse en retour. C'est l'action poursuivie dans la version de Rum and Coca-Cola, chantée par Lord Invador à Trinidad en 1950, lorsqu'il ironise sur les mots des Andrews Sisters tels qu'ils étaient articulés. Une dernière particularité de la structure dialogique des chansons concerne la « citation ». Dans le cas qui nous intéresse, la situation liminale des visiteurs ou immigrés trinidadiens à New York leur permettait de relâcher l'une des règles suivies à Trinidad, où tout calypso est une chanson originale, composée par son chanteur. Lord Invador ne la respectait pas toujours (Masquelier, 2012) ; ce qui est le cas pour son calypso intitulé Tie-tongue baby dont l'enregistrement new-yorkais et son interprétation scénique au Town Hall sont des versions réécrites du calypso originellement composé par Lord Kitchener, à Trinidad, pour le carnaval de 1946. Aussi faudrait-il mentionner, à propos de Rum and Coca-Cola, la situation incongrue en marge de la dispute autour de

39. Ce que je nomme une trame est généralement analysé par les théoriciens de la littérature ou de l'analyse de discours en termes d'intertextualité. 
l'enregistrement des Andrews Sisters : Wilmoth Houdini, émigré trinidadien à New York, compositeur et interprète de calypsos, a enregistré sa propre version de Rum and Coca-Cola en 1945 chez Decca. La mélodie est identique à celle de la version chantée par les Andrews Sisters (ou de celle de Lord Invador). Des dix strophes chantées par Houdini, trois calquent les paroles des Andrews Sisters. Pour les sept autres strophes, l'une invite, non sans humour, les Andrews Sisters à se rendre à Trinidad pour y écouter les chanteurs de calypso au « clair de lune argenté » (under the silvery moon). Les six autres traitent de l'opulence nouvelle que connaît Trinidad grâce à l'argent (money in the land, the yankee dollar bill), de ce qu'il permet d'acquérir (spending money on luxury), des plaisirs qu'il facilite. Houdini concourt à l'idée que Trinidad est un paradis (Trinidad is paradise) sur terre (you will have money and a nice girl friend) : il l'associe aux fêtes alcoolisées et mentionne les ébats amoureux (love making under the bamboo tree) qu'on observe sur les plages de Manzanilla (sur la côte est de Trinidad). Le refrain de ce calypso est chaque fois semblable à celui de la version chantée par les Andrews Sisters. Toutefois, en accord avec la façon de parler l'anglais de Trinidad, Houdini substitue coco cola à Coca-Cola.

Forment en effet un même ensemble toutes les «textualisations » : celles de la chanson enregistrée en studio, celles scénarisées et spontanées des performances scéniques, mais aussi celles des citations par des êtres de fiction (qui ressemblent étrangement aux êtres de «chair et de sang » des faubourgs de Port of Spain, d'hier et d'aujourd'hui), ainsi que les fictions littéraires qui explorent plus longuement les situations évoquées par les calypsos. L'entrée dans cet ensemble ouvert peut se faire aussi bien par l'interprétation que les Andrews Sisters délivrent de leur Rum and Coca-Cola, que par d'autres voies. D'une certaine manière, ce que ces différentes versions disent, pour un auditeur, ou un lecteur, est incomplet, puisque ces derniers peuvent toujours poursuivre leur enquête et étendre le champ de leur compréhension (esthétique, morale et politique) de ce dont il est parlé.

Il faut noter que cette trame associe des moments et des lieux de paroles (ou de musique) et les espaces sociaux particuliers dont ils participent : théâtres et clubs new-yorkais, studios d'enregistrement, cour de justice, bars-théâtres (calypso-tents) et rhumeries de Port of Spain, Trinidad. Par le jeu de métaphores, de métonymies, de citations, de commentaires, cette trame est enchâssée dans l'espace de production et de réception d'œuvres littéraires. Ces traces, celles des écrits comme des performances scéniques, et leurs « chronotopes » (leurs paramètres temporels et spatiaux) font surface ici et là, dans le temps présent. C'est l'expérience que fait le lecteur de Miguel Street par V. Naipaul ou de Rum and Coca-Cola par $\mathrm{R}$. de Boissière - des écrits-mémoires intimement liés à la situation diasporique de leurs auteurs et aux contextes historiques des années 1940 dans les Caraïbes. Ces 
traces participent parfois de la configuration occasionnelle de nouvelles situations d'échange : l'événement rapporté qui introduit cet essai tentait de le montrer.

Deux autres commentaires compléteront ceux qui précèdent. Ils sont établis, ceux-là, du point de vue d'une anthropologie poétique, autrement dit d'une anthropologie linguistique attachée à prendre en compte la dimension expressive du discours (Bauman, 1986). Dans cette perspective, sont particulièrement riches d'enseignement les traces que nous laissent les enregistrements des performances de Lord Invador à New York en 1946 et à Port of Spain en 1950. Elles dévoilent l'émotion du chanteur. L'émotion affleure autant dans ce qu'il veut dire (la dimension argumentative des paroles chantées), que dans leur tonalité, la manière dont il les délivre.

Lord Invador, sur ses scènes, s'indigne de la situation qu'il décrit dans son Rum and Coca-Cola. L'énonciation passionnée des paroles s'incarne dans l'expression de la voix, le tempo du chant, les procédés stylistiques choisis dans l'évocation des situations rapportées. En bref, l'indignation émane de la performance. Elle amplifie la force de conviction et d'argumentation de ce qui est dit, sans trop en dire, des situations décrites par le calypso. L'indignation exprimée sollicite l'attention et la participation de la communauté des auditeurs présents lors de la performance. Au contraire, la version des Andrews Sisters semble être désincarnée, déterritorialisée, et abstraite de tout engagement émotionnel « réel ». Les situations représentées dans le calypso des Andrews Sisters paraissent semblables à celles des « publicités » de papier glacé des agences de tourisme : elles font écran aux événements que les mots profilent. Elles en disent si peu, qu'on ne peut même pas suggérer que ce calypso respecte le goût trinidadien pour l'indirection, l'énigme et le non-dit que l'auditeur trinidadien attend d'un bon calypso dans le moment même de la performance (Masquelier, 2011). Comment ironiser sur ce calypso d'un point de vue trinidadien ? Sinon en ciblant, comme le fait Lord Invador, l'étrange manière des Andrews Sisters de prononcer l'anglais et d'énoncer le nom de Coca-Cola.

Lord Invador s'indigne dans ses chansons des conséquences sociales de la présence nord-américaine dans cette période des années 1940-1950. Il faut alors reconnaître que les écrivains trinidadiens, grâce à leur talent et d'autres capacités, ont prolongé avec à-propos l'exploration des thèmes évoqués dans les calypsos de l'époque et les conversations qui se tenaient dans les bars à rhum. Les quelques références littéraires mentionnées dans cet essai anticipent la littérature postcoloniale, littérature critique de l'hégémonie des puissances coloniales, comme des complexités sociales que les situations coloniales auront produites (Bhabha, 2007). 


\section{Bibliographie}

Bauman Richard, 1986, Story, Performance and Event: Contextual Studies of Oral Narrative, New York: Cambridge University Press.

Bнавна Homi, 2007, les Lieux de la culture : une théorie postcoloniale, Paris : Éditions Payot [1994].

Calame Claude, 2015, Qu'est-ce que la mythologie grecque?, Paris : Gallimard.

Calame Claude, Dupont Florence, Lortat-Jacob Bernard, Manca Maria (dir.), 2010, la Voix actée : pour une nouvelle ethnopoétique, Paris : Éditions Kimé.

De Boissiere Ralph, 1981, Crown Jewel, Lodon: Picador - Pan Books [1952, first published in Australia].

De Boissiere Ralph, 1984, Rum and Coca-Cola, revised edition, London: Allison and Busby Limited [1956, Melbourne, by Australian Book Society].

EldRIDGE Michael, 2002, “There Goes the Transnational Neighborhood: Calypso Buys a Bungalow”, Callaloo, vol. 25, nº 2, p. 620-638.

Erb Melanie, 2009, “G. I. Jane Joins the Band: WAC and the Andrews Sisters”, Vanderbilt Undergraduate Research Journal, vol. 5, p. 1-7.

Hill Donald, 1993, Calypso Calaloo: Early Carnival Music in Trinidad, Gainesville: University Press of Florida.

Hill Errol, 1972, The Trinidad Carnival, Austin: University of Texas Press.

Liverpool Hollis, 2001, Rituals of Power and Rebellion: The Carnival Tradition in Trinidad and Tobago 1763-1962, Chicago: Research Associates School Times Publications [1993].

Masquelier Bertrand, 2011, « Dire et Chanter des mots qui fâchent », Cabiers de littérature orale, $\mathrm{n}^{\circ} 70$, p. 87-117. 
Masquelier Bertrand, 2012, «Goffman et l'Ethnographie des façons de parler : s'excuser d'une fausse note sur la scène du Town Hall de New York, un soir de décembre 1946 », in Daniel CéfaÏ et Laurent Perreau (dir.), Goffman et l'Ordre de l'interaction, Paris - Amiens : CURAPP-ESS/CEMSIMM, p. 427-449.

Masquelier Bertrand, 2014, «The Performative Turn : les nouvelles frontières de l'anthropologie sociale et linguistique », in Sandra Bornand et Cécile Leguy (dir.), Compétence et Performance: perspectives interdisciplinaires sur une dichotomie classique, Paris : Karthala, p. 306-329.

Naipaul Vidia S., 1967, Miguel Street, Paris : Gallimard [1959].

Neptune Harvey, 2007, Caliban and the Yankees: Trinidad and the United-States Occupation, Chapel Hill: University of North Carolina Press.

Nizer Louis, 1961, My Life in Court, New York: Doubleday \& Co.

Ochs Elinor, 1979, "Transcription as Theory", in Elinor OcHs and Bambi Schieffelin (ed.), Developmental Pragmatics, New York: Academic Press, p. 43-72.

Silverstein Michael, Urban Gregg (ed.), 1996, Natural Histories of Discourse, Chicago: University of Chicago Press.

\section{Discographie}

Belasco Lionel, Goodnight Ladies and Gents, Annotation by Donald R. Hill, 1999, Rounder Records no 1138.

Calypso at Midnight, vol. 1: Concert and Radio Series (Lomax collection), edited and with notes by Donald R. Hill and John H. Cowley, preface by S. Shapiro, 1999, Rounder Records no 1840. [Lord Invador, Rum and Coca-Cola, 1946]

Calypso after Midnight, vol. 2: Concert and Radio Series (Lomax collection), edited and with notes by Donald R. Hill and John H. Cowley, Preface by 
S. Shapiro, 1999, Rounder Records n ${ }^{\circ}$ 1841. [Lord Invador, The G.I and the Lady, Yankee Dollar, 1946]

Calypso Calaloo, notes by Donald R. Hill, Various Artists, Rounder Records $\mathrm{n}^{\circ}$ 1105. [Lord Invador, Rum and Coca-Cola, 1950]

Calypso 1940-1952, Conceived and compiled by Jean-Michel Gibert, 2004, GMC Ltd. [Andrews Sisters, Rum and Coca-Cola, 1944]

Don't Stop the Carnival, Trinidad and Calypso, Records from the collection David Lennick, Colin Bray, John Rutherford, Recording Archives/ University of Toronto and the Belfer Audio Laboratory and Archive, Syracuse University Library, annotated by Tony WATTS, 1998, PAST CD 7825.[Lord Invador, The Soldiers Came and Broke up my Life, 1945 ; Wilmoth Houdini, Rum and Coca-Cola, 1945]

Lord Invador Calypso in New York, The Asch Recordings 1946-1961, annotated by John Cowley, 2000, Smithsonian Folkways Recordings no 40454. [Lord Invador, Tie-Tongue Baby, 1946]

Trinidad-Calypso : 1939-1959, livret texte de Bruno Blum, Frémeaux \& associés, 2011, FA5348. [Lord Invador, Rum and Coca-Cola, 1950]

Résumé : Les chanteurs de calypso (calypsonians) jouent un rôle public de premier plan à Trinidad. C'est toujours le cas aujourd'hui, bien que les styles musicaux et textuels du calypso changent. Dans le contexte local, ces chanteurs, lorsqu'ils commentent les affaires politiques du moment, se présentent comme les porte-paroles du peuple. Une manière de participer au débat public à Trinidad consiste donc à mettre en chanson ce que l'on souhaite dire. Qu'en est-il lorsqu'un calypso, dans une version remaniée, est approprié et interprété, hors contexte, par des chanteurs européens ou nord-américains pour un public étranger ? L'article explore une telle situation. Il montre comment ce que signifie un calypso fait écho, à travers le temps et l'espace, à d'autres performances et d'autres versions. Il faut aussi compter avec la manière dont les auteurs de la littérature caribéenne se sont appropriés cette manière de parler.

Mots clés : Caraïbes, Trinidad, calypso, littérature, performance, débat public, espace public 


\section{CAHIERS DE LITTÉRATURE ORALE}

224 Paroles publiques, paroles confidentielles $-\mathrm{n}^{\circ}$ 77-78

Abstract: Calypsonians play an important public role in Trinidad. They did in the past, as they do today, even though the musical and textual styles of calypsos have changed over the years. Locally, calypsonians claim to be the spokespersons of the people (of Trinidad), especially when their songs deliver commentaries on current (local or global) political issues. From this perspective, one way of being an active participant in the debates of the public sphere is to sing calypsos. What happens when a calypso, or its revised copy, is sung by European or North American singers for a foreign audience? This paper discusses such a situation. It shows that the significance of a particular song depends on the set of other songs and performances to which it belongs in time and space, and that one should also take into account how literary figures (writers) of Caribbean literature have fictionalized calypsos.

Keywords: Caribbean, Trinidad, Calypso, Literature, Performance, Public Debate, Public Space 